\title{
Decomposition dynamics of interstellar HCNH: Ab-initio MO and RRKM studies
}

\author{
H. Tachikawa ${ }^{1}$, T. Iyama ${ }^{1}$, and T. Fukuzumi ${ }^{2}$ \\ 1 Division of Molecular Chemistry, Graduate School of Engineering, Hokkaido University, Sapporo 060-8628, Japan \\ 2 Nakanoshima Astronomical Observatory, Takao, Nakanoshima, Toshima, 891-5201, Japan
}

Received 23 April 2002 / Accepted 12 September 2002

\begin{abstract}
Ab-initio MO calculations have been carried out for hydrogen-dissociation reactions $\mathrm{HCNH}$, i.e. $\mathrm{HCNH} \rightarrow \mathrm{H}+$ $\mathrm{HCN}$ (I) and $\mathrm{HCNH} \rightarrow \mathrm{HNC}+\mathrm{H}$ (II), in order to elucidate the branching ratio of $\mathrm{HCN} / \mathrm{HNC}$ on the ground state potential energy surface. The calculations showed that the transition state for reaction I is lower in energy than that of reaction II. The "bare" barrier heights for channel I and II were calculated to be $33.5-34.8 \mathrm{kcal} / \mathrm{mol}$ and $38.5-40.7 \mathrm{kcal} / \mathrm{mol}$, respectively. The energy difference between transition states I and II was calculated to be $3.7-6.9 \mathrm{kcal} / \mathrm{mol}$, meaning that reaction I preferentially occurs in the threshold energy region. Rice-Ramsperger-Kassel-Marcus (RRKM) theory including the tunneling effect indicated that reaction I is more favorable than reaction II at lower energy region, if tunneling effects are included in the rate calculations. On the other hand, the higher energy region above ca. $50 \mathrm{kcal} / \mathrm{mol}$, channel II became dominant. The branching ratio (HCN/HNC) was calculated to be 0.3 at $E=4.4 \mathrm{eV}$, which corresponds to the electron affinity of $\mathrm{HCNH}^{+}$. The mechanism of the reaction is discussed on the basis of theoretical results.
\end{abstract}

Key words. molecular processes - ISM: molecules - molecular data

\section{Introduction}

$\mathrm{HCN}, \mathrm{HNC}$, and HCNH molecules are important in the chemical evolution process in interstellar molecular clouds because these molecules are considered as initial species in the synthesis of amino acid and protein (Suzuki et al. 1992). Study of the formation mechanism would provide knowledge of early stages of chemical evolution. $\mathrm{HCN}$ and $\mathrm{HNC}$ molecules have been considered to be formed by electron capture of $\mathrm{HCNH}^{+}$ as following reactions (Talbi et al. 1996),

$$
\begin{aligned}
\mathrm{HCNH}^{+}+\mathrm{e}^{-} \rightarrow[\mathrm{HCNH}]^{*} & \rightarrow \mathrm{HNC}+\mathrm{H} \\
& \rightarrow \mathrm{HCN}+\mathrm{H}
\end{aligned}
$$

where $[\mathrm{HCNH}]^{*}$ is the neutral $\mathrm{HCNH}$ molecule with $1^{2} \Sigma^{+}$, $2^{2} \Sigma^{+}$, and $1^{2} \Pi$ electronic states or Rydberg states. The interaction between $\mathrm{HCNH}^{+}$and electrons are composed of an attractive Coulomb force, so that a large cross-section is expected. This means that electron capture of $\mathrm{HCNH}^{+}$proceeds efficiently. Also, $\mathrm{HCNH}^{+}$is known to be a stable ion that does not react with large number of hydrogen molecules existing in interstellar molecular clouds. Hence, $\mathrm{HCNH}^{+}$exists as a longlived molecular ion in the clouds and is a major candidate in the formation of $\mathrm{HCN}$ and $\mathrm{HCN}$ molecules.

The HCN/HNC ratios in molecular clouds are often observed to be 1 . From ab-initio molecular orbital (MO)

Send offprint requests to: Dr. H. Tachikawa,

e-mail: hiroto@eng.hokudai.ac.jp calculations of the Franck-Condon (FC) region, this ratio was supported theoretically (Shiba et al. 1998; Herbst 1979; Talbi \& Ellinger 1998, 1996). It is suggested that the $\mathrm{H}$ dissociation from HCNH occurs mainly on the $1^{2} \Sigma^{+}$and $2^{2} \Sigma^{+}$state surfaces. Thus, the hydrogen dissociations from $\mathrm{HCNH}$ at $1^{2} \Sigma^{+}$ and $2^{2} \Sigma^{+}$states were well understood theoretically. However, the decomposition of $\mathrm{HCNH}$ on the ground state potential energy surface $\left(\mathrm{X}^{2} \mathrm{~A}^{\prime}\right.$ in Cs symmetry or $\mathrm{X}^{2} \Pi$ in $C_{\infty \mathrm{v}}$ symmetry) is not clearly understood.

In the present study, ab-initio MO calculations are carried out for the hydrogen dissociation reactions from $\mathrm{HCNH}$ on the ground state potential energy surface (PES). Also, the Rice-Ramsperger-Kassel-Marcus (RRKM) theory is applied to the reaction system in order to obtain the branching ratio of HCN and HNC. This PES corresponds to that of a hydrogen exchange reaction $\mathrm{H}+\mathrm{CNH} \rightarrow \mathrm{HCN}+\mathrm{H}$, which is also important in the formation of the $\mathrm{HCN}$ molecule. This work could provide important information for the origin of amino acids and protein in the Universe.

\section{Computational methods}

All ab-initio molecular orbital (MO) calculations were carried out using the Gaussian 98 program package (Frisch et al. 1998). The geometry optimizations of molecules at the stationary points were made at the Møller-Plesset forth order perturbation theory with Dunning's correlation consistent basis set (valence triple-zeta): namely, MP4SDQ /cc-pVTZ level. 


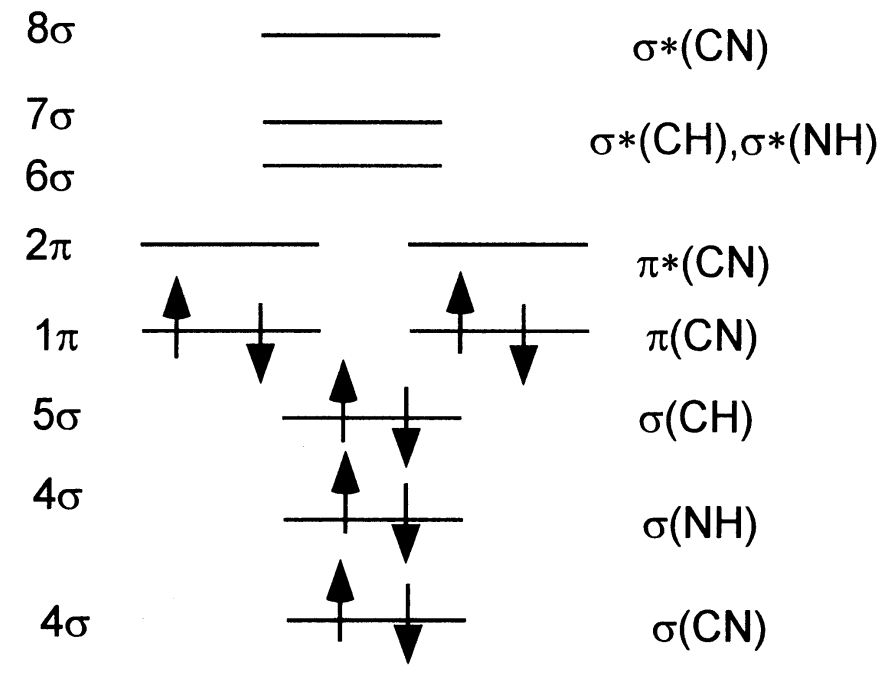

Fig. 1. Schematic illustration for molecular orbitals of $\mathrm{HCNH}^{+}$.

Also, the MP4SDQ/6-311++G(d, p) calculation was carried out in order to obtain the geometry of the molecules. Using the optimized geometries, the energies were re-calculated by the quadratic configuration interaction including the single and double substitution (QCISD) calculation with standard Pople's basis sets: namely, the QCISD/6-311++G(2df, 2pd) and QCISD/6-311++G(3df, 3pd) levels. Also, total energies were calculated at the QCISD/aug-cc-pVTZ levels.

The electronic configuration of $\mathrm{HCNH}^{+}$is illustrated in Fig. 1.

The electrons occupy up to the double degerated $\pi \mathrm{CN}$ ) molecular orbital $(1 \pi$ orbital $)$ of $\mathrm{HCNH}^{+}$. If an excess electron occupies $\pi^{*}(\mathrm{CN})$ orbital $(2 \pi$ rbital) by electron capture, $\mathrm{HCNH}^{+}+\mathrm{e}^{-} \rightarrow \mathrm{HCNH}$, the electronic state of $\mathrm{HCNH}$ is expressed by the ${ }^{2} \Pi$ state. This is the ground state of HCNH. $\mathrm{HCNH}$ has an excited state expressed by $\mathrm{HCNH}\left({ }^{2} \Sigma\right)$ if the excess electron occupies in the $\sigma^{*}(\mathrm{CH})$ orbital. In the present study, we considered only the ${ }^{2} \Pi$ state of $\mathrm{HCNH}$ (i.e., the ground state of $\mathrm{HCNH}$ ). This state is changed to ${ }^{2} \mathrm{~A}^{\prime}$ state if the molecular symmetry is reduced to the Cs symmetry.

Harmonic vibrational frequencies of the molecules at the stationary points along the reaction coordinates, HCNH, TS1, TS2, HCN and HNC, were calculated at the MP4SDQ/6$311++\mathrm{G}(\mathrm{d}, \mathrm{p})$ level. In addition, in order to estimate isotope effect on the reaction, the harmonic frequencies for the systems including deuterium, DCND, DCNH and HCND, were calculated with the same manner.

The reaction rates were calculated using RRKM theory as a function of excess energy of HCNH. In the RRKM calculations, the tunneling effect was included by Miller's method (Miller 1979)

\section{Results}

\section{A. Structures and energetics of the $\mathrm{HCNH}$ system}

The present calculations showed that two reaction channels are involved in the hydrogen atom dissociations from $\mathrm{HCNH}$ on the ground state potential energy surface $\left({ }^{2} \Pi\right.$ or ${ }^{2} \mathrm{~A}^{\prime}$ states $)$ expressed by

$$
\begin{aligned}
\mathrm{HCNH}\left({ }^{2} \Pi\right) & \rightarrow \mathrm{TS} 1 \rightarrow \mathrm{HCN}+\mathrm{H} \text { channel I } \\
& \rightarrow \mathrm{TS} 2 \rightarrow \mathrm{HNC}+\mathrm{H} \text { channel II. }
\end{aligned}
$$

Channels I and II are pathways for the $\mathrm{N}-\mathrm{H}$ and $\mathrm{C}-\mathrm{H}$ bond cleavage reactions of $\mathrm{HCNH}$, respectively. Each channel has a transition state expressed by TS1 or TS2. The structures of $\mathrm{HCNH}$ at the stationary points along the $\mathrm{H}$-atom dissociation reactions (channels I and II) were fully optimized at the MP4SDQ/cc-pVTZ and MP4SDQ/6-311++G(d, p) levels of theory. Both calculations gave similar geometrical parameters. The optimized structures obtained at the MP4SDQ/cc-pVTZ level are illustrated in Fig. 1. The HCNH molecule has a bent structure with a trans form and a planar one $\left(\phi=180^{\circ}\right)$. The structures of TS1 and TS2 are also illustrated in Fig. 2. Both transition states have a cis form. The product molecules for channels I and II are $\mathrm{HCN}$ and $\mathrm{HNC}$, respectively. The bond distances of $\mathrm{HCN}, r(\mathrm{C}-\mathrm{H})$ and $r(\mathrm{CN})$, were calculated to be 1.0648 and $1.1551 \AA$, respectively, in good agreement with experimental values (1.065 and $1.153 \AA$ ). For the HNC molecule, a reasonable structure was also obtained. These results strongly indicate that the MP4SDQ/cc-pVTZ calculation would give reasonable structures for the $\mathrm{HCNH}$ system.

The total energies and potential energy curve calculated at the MP4SDQ/cc-pVTZ, QCISD/aug-cc-pVTZ, MP4SDQ/aug-cc-pVTZ, QCISD/6-311++G(2df, 2pd), and QCISD/6-311++G(3df, 3pd) levels are given in Fig. 3. The structure at each stationary point was fully optimized at the MP4SDQ/cc-pVTZ level. Relative energies are given in Table 1. The calculations indicate that the barrier heights for channels I and II are close to each other, although that of channel II was always lower than that of channel I in all levels.

Harmonic vibrational frequencies of $\mathrm{HCNH}, \mathrm{TS} 1$, TS2, HCN and HNC were calculated at the MP4SDQ/6$311++\mathrm{G}(\mathrm{d}, \mathrm{p})$ level. The results are summarized in Table 2. Inclusion of zero-point vibrational energies changed relative energies to the low-energy region. However, TS1 is always lower in energy than that of TS2.

For comparison, vibrational frequencies of the molecules at the stationary points for the deuterium dissociation reactions,

$$
\begin{aligned}
\mathrm{DCND}^{+}\left({ }^{2} \Pi\right)+\mathrm{e}^{-} & \rightarrow[\mathrm{DCND}]^{*} \\
& \rightarrow \mathrm{TS} 1(\mathrm{D}) \rightarrow \mathrm{DNC}+\mathrm{D} \text { channel I(D) } \\
& \rightarrow \mathrm{TS} 2(\mathrm{D}) \rightarrow \mathrm{DCN}+\mathrm{D} \text { chennel II(D) }
\end{aligned}
$$

were calculated in the same manner. The results are given in Table 3. The stretching modes of DCND (modes 1-3) were largely shifted to low-energy region. The imaginary frequencies at the transition states TS1(D) and TS2(D) were calculated to be $1337.1 i$ and $683.5 i \mathrm{~cm}^{-1}$, respectively, which are more blue-shifted than that of HCNH $\left(1801.9 i\right.$ and $\left.931.8 i \mathrm{~cm}^{-1}\right)$. The zero point vibrational energies (ZPEs) were lowered by the isotope effect. The vibrational frequencies of the molecules at the stationary points for the HCND and DCNH were also calculated and the results are summarized in Table 4. The imaginary frequencies for the $\mathrm{D}$ atom dissociation were lower than that of hydrogen. 


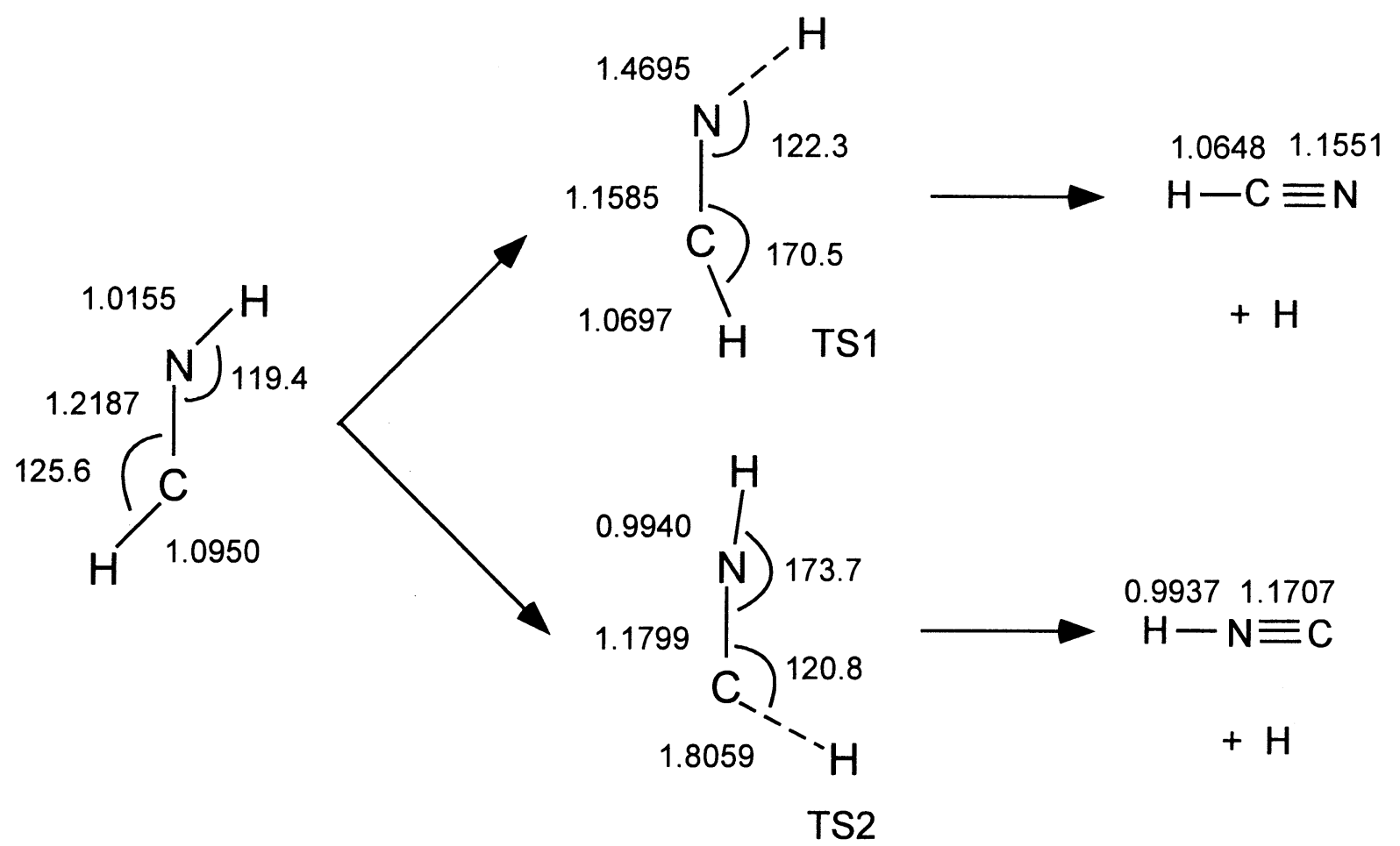

Fig. 2. Optimized structures of HCNH system. The values were calculated at the MP4SDQ/cc-pVTZ level. Bond lengths and angles are in $\AA$ and in degrees, respectively. Experimental geometrical parameters are $r(\mathrm{CH})=1.065$ and $r(\mathrm{CN})=1.153 \AA$ for HCN (Herzberg 1966), and $r(\mathrm{NH})=0.986$ and $r(\mathrm{CN})=1.173 \AA$ for HNC (Harmony et al. 1979).

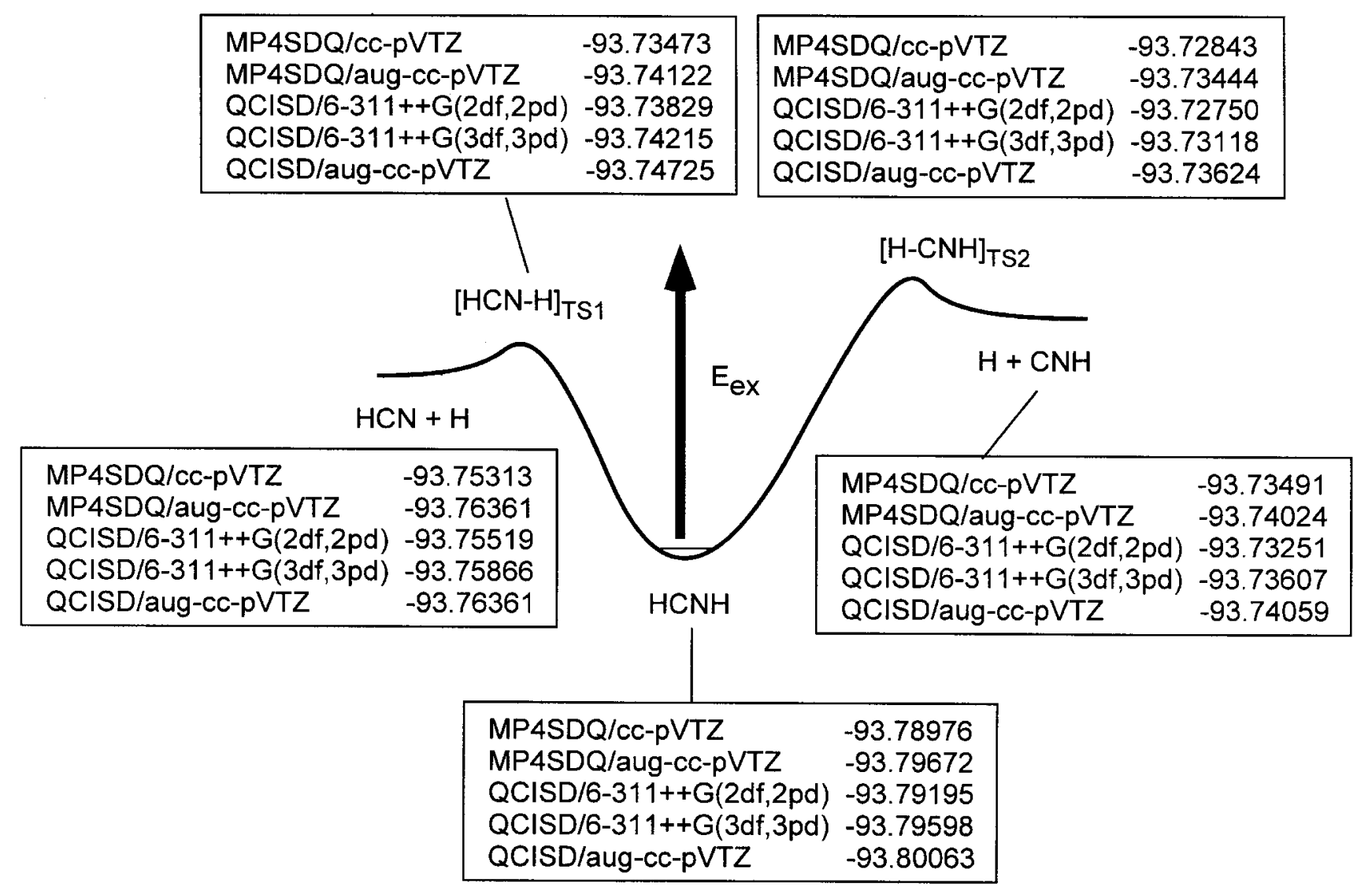

Fig. 3. Potential energy curve for the hydrogen atom decomposition of HCNH calculated for several levels. The structures at each stationary point were obtained at the MP4SDQ/cc-pVTZ level (see Fig. 1). 
Table 1. Relative energies $\left(E_{\text {rel }}\right.$ in $\left.\mathrm{kcal} \mathrm{mol}^{-1}\right)$ calculated at various levels ${ }^{a}$.

\begin{tabular}{llllll}
\hline \hline & HCNH & TS1 & TS2 & HCN + H & HNC + H \\
\hline MP4SDQ/cc-pVTZ & 0.0 & 34.8 & 38.5 & 23.0 & 34.4 \\
MP4SDQ/aug-cc-pVTZ & 0.0 & 34.8 & 39.1 & 20.8 & 35.4 \\
QCISD/6-311++G(2df,2pd) & 0.0 & 33.7 & 40.4 & 23.1 & 37.3 \\
QCISD/6-311++G(3df,3pd) & 0.0 & 33.8 & 40.7 & 23.4 & 37.6 \\
QCISD/aug-cc-pVTZ & 0.0 & 33.5 & 40.4 & 23.2 & 37.7 \\
\hline
\end{tabular}

${ }^{a}$ Geometries were fully optimized at the MP4SDQ/cc-pVTZ level.

Table 2. Harmonic vibrational frequencies calculated at the MP4SDQ/6-311++G(d, p) level $\left(\mathrm{in}^{-1}\right)$. Zero point vibrational energies $(Z P E$ in $\mathrm{kcal} / \mathrm{mol})$ and best estimated relative energies $\left(E_{\text {rel }}+\triangle Z P E\right)$ are also given. $E_{\text {rel }}$ were calculated at the QCISD/aug-cc-pVTZ//MP4SDQ/ccpVTZ level.

\begin{tabular}{llllll}
\hline \hline & HCNH & TS1 & TS2 & HCN $^{a}+\mathrm{H}$ & HNC $^{b}+\mathrm{H}$ \\
\hline 1 & $3541.1\left(\mathrm{a}^{\prime}\right)$ & $1801.9 \mathrm{i}\left(\mathrm{a}^{\prime}\right)$ & $931.8 \mathrm{i}\left(\mathrm{a}^{\prime}\right)$ & $3475.9(\sigma)$ & $3860.9(\sigma)$ \\
2 & $3070.3\left(\mathrm{a}^{\prime}\right)$ & $3491.0\left(\mathrm{a}^{\prime}\right)$ & $3853.7\left(\mathrm{a}^{\prime}\right)$ & $2121.5(\sigma)$ & $2053.3(\sigma)$ \\
3 & $2246.3\left(\mathrm{a}^{\prime}\right)$ & $2359.6\left(\mathrm{a}^{\prime}\right)$ & $2043.5\left(\mathrm{a}^{\prime}\right)$ & $739.6(\pi)$ & $484.5(\pi)$ \\
4 & $1203.5\left(\mathrm{a}^{\prime}\right)$ & $881.9\left(\mathrm{a}^{\prime \prime}\right)$ & $518.9\left(\mathrm{a}^{\prime \prime}\right)$ & $739.6(\pi)$ & $484.5(\pi)$ \\
5 & $982.5\left(\mathrm{a}^{\prime \prime}\right)$ & $774.2\left(\mathrm{a}^{\prime}\right)$ & $423.8\left(\mathrm{a}^{\prime}\right)$ & & \\
6 & $859.8\left(\mathrm{a}^{\prime}\right)$ & $559.7\left(\mathrm{a}^{\prime}\right)$ & $355.3\left(\mathrm{a}^{\prime}\right)$ & & \\
$Z P E$ & 17.0 & 11.6 & 10.3 & 10.1 & 9.8 \\
$E_{\text {rel }}+\Delta Z P E$ & 0.0 & 28.1 & 33.7 & 16.3 & 30.5 \\
\hline
\end{tabular}

${ }^{a}$ Experimental values were measured to be $3311.5,2096.9$ and $712.0 \mathrm{~cm}^{-1}$ (Shimanouchi et al. 1992).

${ }^{b}$ Experimental values were measured to be 3652, 2023.9 and $464.2 \mathrm{~cm}^{-1}$ (Pettersson 1998).

Table 3. Harmonic vibrational frequencies of the DCND system calculated at the MP4SDQ/6-311++G(d, p) level (in $\left.\mathrm{cm}^{-1}\right)$. Zero point vibrational energies $(Z P E$ in $\mathrm{kcal} / \mathrm{mol})$ and best estimated relative energies $\left(E_{\text {rel }}+\triangle Z P E\right)$ are also given. $E_{\text {rel }}$ was calculated at the QCISD/augcc-pVTZ//MP4SDQ/cc-pVTZ level.

\begin{tabular}{llllll}
\hline \hline & DCND & TS1(D) & TS2 $(\mathrm{D})$ & DCN + D & DNC + D \\
\hline 1 & $2642.0\left(\mathrm{a}^{\prime}\right)$ & $1337.1 \mathrm{i}\left(\mathrm{a}^{\prime}\right)$ & $683.5 \mathrm{i}\left(\mathrm{a}^{\prime}\right)$ & $2723.3(\sigma)$ & $2905.7(\sigma)$ \\
2 & $2399.2\left(\mathrm{a}^{\prime}\right)$ & $2816.8\left(\mathrm{a}^{\prime}\right)$ & $2899.7\left(\mathrm{a}^{\prime}\right)$ & $1950.6(\sigma)$ & $1964.8(\sigma)$ \\
3 & $2039.3\left(\mathrm{a}^{\prime}\right)$ & $2075.2\left(\mathrm{a}^{\prime}\right)$ & $1955.4\left(\mathrm{a}^{\prime}\right)$ & $589.7(\pi)$ & $383.2(\pi)$ \\
4 & $954.8\left(\mathrm{a}^{\prime}\right)$ & $718.7\left(\mathrm{a}^{\prime \prime}\right)$ & $410.1\left(\mathrm{a}^{\prime \prime}\right)$ & $589.7(\pi)$ & $383.2(\pi)$ \\
5 & $720.7\left(\mathrm{a}^{\prime \prime}\right)$ & $639.7\left(\mathrm{a}^{\prime}\right)$ & $353.6\left(\mathrm{a}^{\prime}\right)$ & & \\
6 & $630.4\left(\mathrm{a}^{\prime}\right)$ & $410.6\left(\mathrm{a}^{\prime}\right)$ & $257.7\left(\mathrm{a}^{\prime}\right)$ & & \\
$Z P E$ & 13.4 & 9.5 & 8.4 & 8.4 & 8.1 \\
$E_{\text {rel }}+\Delta Z P E$ & 0.0 & 29.6 & 35.4 & 18.2 & 32.4 \\
\hline
\end{tabular}

\section{B. Reaction rates}

On the basis of the energetics and vibrational frequencies, reaction rates for channels I and II were calculated as a function of internal energy $(E)$ of HCNH using RRKM theory including tunneling effects. The results are given in Fig. 3. At lower internal energy, channel I was significantly dominant. The curves of reaction rates for channel I and II crossed each other at $E=48 \mathrm{kcal} / \mathrm{mol}$. Channel II becomes dominant at higher energies. At $E=4.4 \mathrm{eV}$, which corresponds to the electron affinity of $\mathrm{HCNH}^{+}$(Shiba et al. 1998), the branching ratio for $\mathrm{HCN} / \mathrm{HNC}$ was calculated to be 0.3 . The corresponding branching ratio for DCN/DNC was calculated to be 2.8 at $E=4.4 \mathrm{eV}$, which is significantly larger than the reaction of the hydrogen atom. This is due to the isotope effect in deuterium.

For comparison, the ratios for the $\mathrm{H}$ or $\mathrm{D}$ atom dissociation reactions of DCNH and HCND were calculated. The ratio of $\mathrm{DNC} / \mathrm{HCN}$ is calculated to be 5.9 for $\mathrm{HCND}$, is 0.63 in $\mathrm{DCNH}$.

\section{Discussion}

\section{A. Summary}

In the present study, the potential energy diagram for the hydrogen dissociation reactions from $\mathrm{HCNH}, \mathrm{HCNH} \rightarrow \mathrm{HCN}+\mathrm{H}$ (I) and $\mathrm{HCNH} \rightarrow \mathrm{HNC}+\mathrm{H}$ (II), were calculated by means of extensive ab-initio MO calculations. The activation barrier for channel I was always lower in energy than that of channel II. The energetics derived form the present calculations are essentially similar to the energy diagram for $\mathrm{H}+\mathrm{CNH} \rightarrow \mathrm{HCN}+\mathrm{H}$ obtained by Talbi et al. (Talbi et al. 1996). Using the energetics data obtained, the reaction rates were calculated on the basis of RRKM theory including tunneling effects. The reaction rates for channel I were larger than those of channel II at lower internal energies of $\mathrm{HCNH}$, below $E=48 \mathrm{kcal} / \mathrm{mol}$, whereas channel II became dominant at higher energy. The branching ratio $\mathrm{HCN} / \mathrm{HNC}$ was estimated to be 0.3 at an internal energy 
Table 4. Harmonic vibrational frequencies of the HCND and HCND systems calculated at the MP4SDQ/6-311++G(d, p) level (in $\left.\mathrm{cm}^{-1}\right)$.

\begin{tabular}{lllllll}
\hline \hline Stationary point & \multicolumn{7}{c}{ frequency } \\
\hline HCND & 3553.6 & 2448.3 & 2058.6 & 1079.1 & 865.6 & 742.8 \\
TS1 & $1353.9 \mathrm{i}$ & 3499.7 & 2328.1 & 891.3 & 744.5 & 452.6 \\
TS2 & $933.9 \mathrm{i}$ & 2907.0 & 1958.1 & 417.3 & 412.0 & 279.4 \\
\multicolumn{7}{c}{ Reaction of DCNH } \\
DCNH & 3083.2 & 2629.8 & 2204.2 & 1153.9 & 863.9 & 685.3 \\
TS1 & $1794.8 \mathrm{i}$ & 2836.5 & 2099.9 & 724.0 & 696.6 & 492.9 \\
TS2 & $688.3 \mathrm{i}$ & 3867.2 & 2042.7 & 519.9 & 390.0 & 309.2 \\
\hline
\end{tabular}

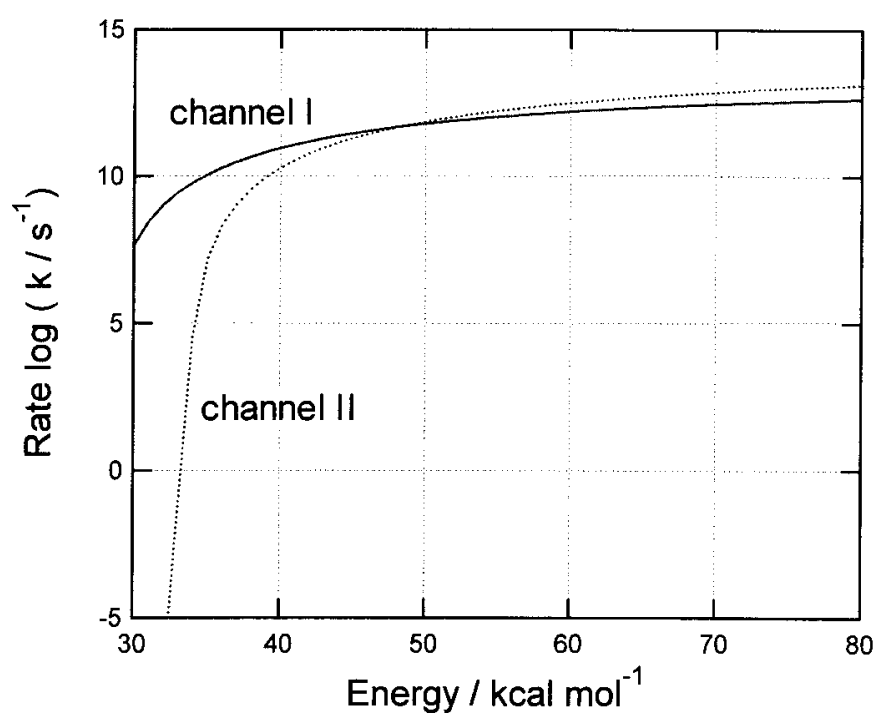

Fig. 4. Reaction rates for channels I and II plotted as a function of internal energy of HCNH calculated by means of RRKM theory including tunneling effects.

of $4.4 \mathrm{eV}$, which corresponds to the adiabatic electron affinity of $\mathrm{HCNH}^{+}$(Shiba et al. 1998).

\section{B. Reaction model}

It is known that the observed abundance ratios of $\mathrm{HCN}$ and $\mathrm{HNC}$ are dependent on the temperature of the molecular clouds. The ratios in OMC-1 range from 1 to 100 (Schilke et al. 1992). Figure 4 shows the observed $\mathrm{HCN} / \mathrm{HNC}$ ratios in various sources plotted against the reciprocal of temperature of the molecular clouds (Hirota et al. 1998). At high temperature, the ratios lie on a straight line. The ratios are not dependent on temperature at lower temperature regions, although the values are widely distributed. It is considered that HNC is destroyed by reactions with the other atoms: $\mathrm{HNC}+\mathrm{H} \rightarrow \mathrm{HCN}+\mathrm{H}$ and $\mathrm{HNC}+\mathrm{O} \rightarrow \mathrm{NH}+\mathrm{CO}$ for example (Schilke et al. 1992; Talbi et al. 1996). These reactions have activation energies and rate constants strongly dependent on temperature.

For a mechanism in the low temperature region, some theoretical calculations have been carried out by several groups (Shiba et al. 1998; Herbst 1979; Talbi \& Ellinger 1998, 1996; Tachikawa 1999). Previous theoretical studies predicted that the branching ratio $\mathrm{HCN} / \mathrm{HCN}$ formed via two ${ }^{2} \Sigma$ states is

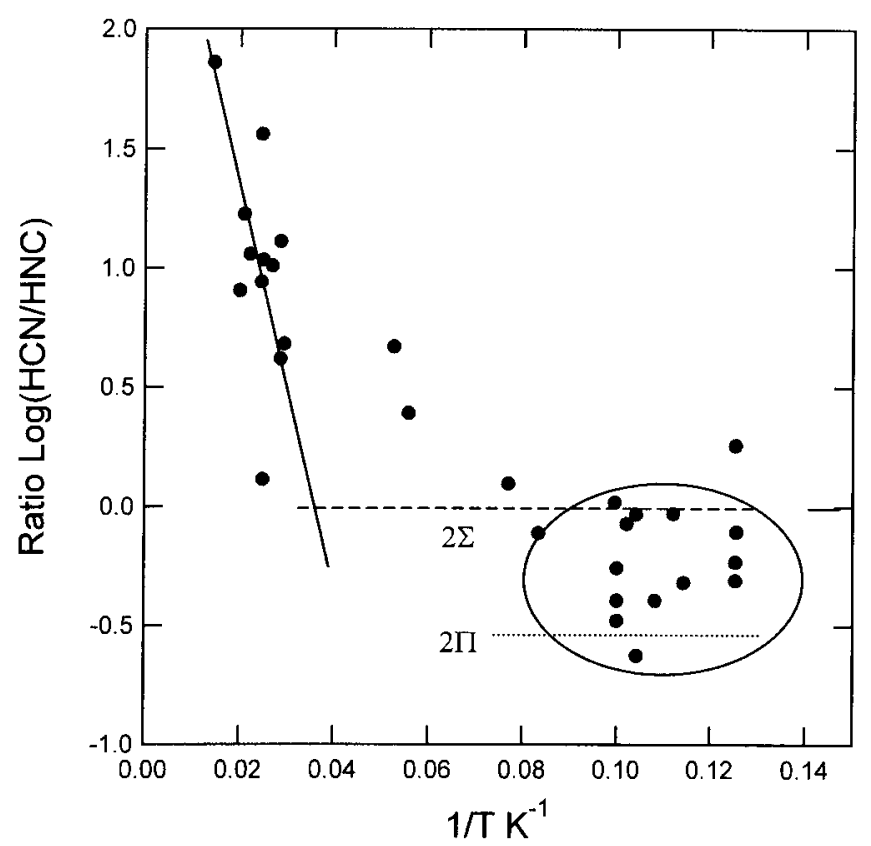

Fig. 5. Observed $\mathrm{HCN} / \mathrm{HNC}$ ratio in various sources is plotted against 1/T. Data are taken from Hirota et al. (1998, ApJ 503, 717). The OMC1 data (Schilke et al. 1992) are fitted to the function $[\mathrm{HCN}] /[\mathrm{HNC}]=$ $A \exp (-\Delta E / T)$, where $\Delta E$ is the potential barrier drawn as a solid line. The dashed line $\left({ }^{2} \Sigma\right)$ is the ratio predicted by the Franck-Condon model via two ${ }^{2} \Sigma$ states. The dotted line $\left({ }^{2} \Pi\right)$ is the ratio predicted by the present model which the reaction occurs via the $\mathrm{HCNH}\left({ }^{2} \Pi\right.$ and ${ }^{2} \mathrm{~A}^{\prime}$ ) state.

closed to 1 . This ratio was calculated on the basis of FranckCondon (FC) model of electron capture of $\mathrm{HCNH}^{+}$with the assumption that the reaction occurs via excited states of $\mathrm{HCNH}$ ( ${ }^{2} \Sigma$ states). This value was schematically illustrated by a dashed line in Fig. 5 (i.e., the line of $\log (\mathrm{HCN} / \mathrm{HNC})=0$, and shown by ${ }^{2} \Sigma$ ). The ratio is not dependent on temperature. This FC model can reasonably explain almost all observed abundance ratios in low temperature. However, as clearly seen in this figure, the observed ratios seem to be widely distributed in the range $\log (\mathrm{HCN} / \mathrm{HNC})=-0.64-0.25$ in lower temperature regions, indicated by a circle in Fig. 5 .

The present value obtained by RRKM theory is schematically plotted by a doted line $\left({ }^{2} \Pi\right.$ state $)$ in Fig. 5 . If a reaction occurs via the ground state potential energy surface, the branching ratio is calculated to be $\mathrm{HCN} / \mathrm{HNC}=0.3$ $(\log (\mathrm{HCN} / \mathrm{HNC})=-0.52$ at $E=4.4 \mathrm{eV})$. This model can 
also explain some observed branching ratios in the circle region in Fig. 4. This agreement may imply that some of the HCN and $\mathrm{HNC}$ molecules are formed via the ground state of $\mathrm{HCNH}\left({ }^{2} \Pi\right.$ state) after the electron capture of $\mathrm{HCNH}^{+}$.

The isotope effects on the branching ratios were also investigated by means of ab-initio and RRKM calculations for the $\mathrm{H}$ and $\mathrm{D}$ dissociation reactions in DCND, HCND and DCNH. The values obtained are consistent with the observed differences in DCN/HCN branching ratios as reported by Turner (2001).

Our main results can be summarized as follows,

(1) In the temperature dependent region, the formation and decomposition of the $\mathrm{HCN}$ and $\mathrm{HNC}$ molecules occurs via $\mathrm{HNC}+\mathrm{H} \rightarrow \mathrm{HCN}+\mathrm{H}$ or $\mathrm{HNC}+\mathrm{O} \rightarrow \mathrm{NH}+\mathrm{CO}$.

(2) In the temperature independent region, in particular the region where the ratio of $\mathrm{HCN} / \mathrm{HNC}$ is close to 1.0 , the electron capture reaction

$$
\begin{aligned}
\mathrm{HCNH}^{+}+\mathrm{e}^{-} \rightarrow\left[\mathrm{HCNH}\left({ }^{2} \Sigma\right)\right]^{*} & \rightarrow \mathrm{HNC}+\mathrm{H} \\
& \rightarrow \mathrm{HCN}+\mathrm{H}
\end{aligned}
$$

may be dominant.

(3) In the temperature independent region, in particular the region where the ratio of $\mathrm{HCN} / \mathrm{HNC}$ is below 1.0, the decomposition reaction on the ground state $\mathrm{PES}$,

$$
\begin{aligned}
\mathrm{HCNH}^{+}+\mathrm{e}^{-} \rightarrow\left[\mathrm{HCNH}\left({ }^{2} \Pi\right)\right]^{*} & \rightarrow \mathrm{TS} 1 \rightarrow \mathrm{HNC}+\mathrm{H} \\
& \rightarrow \mathrm{TS} 2 \rightarrow \mathrm{HCN}+\mathrm{H}
\end{aligned}
$$

would be dominant. Thus, the present model could explain the relation between the branching ratio $(\mathrm{HNC} / \mathrm{HCN})$ and temperature.
Acknowledgements. The authors are indebted to the Computer Center at the Institute for Molecular Science (IMS) for the use of the computing facilities. H.T. also acknowledges a partial support from a Grantin-Aid from the Ministry of Education, Science, Sports and Culture of Japan.

\section{References}

Frisch, M. J., Trucks, G. W., Schlegel, H. B., et al. 1998, Ab-initio MO calculation program Gaussian 98, Revision A.9 (Gaussian, Inc., Pittsburgh PA)

Harmony, M. D., Laurie, V. W., Kuckzowski, R. L., et al. 1979, J. Phys. Chem. Ref. Data, 8, 619

Herzberg, G. 1966, Electronic Spectra and Electronic Structures of Polyatomic Molecules (Van Nostrand, New York)

Hirota, T., Yamamoto, S., Mikami, H., \& Ohishi, M. 1998, ApJ, 503, 717

Miller, W. H. 1979, J. Am. Chem. Soc., 101, 6810

Schilke, P., Walmsley, C. M., Pineau des Forêts, G., et al. 1992, A\&A, 256, 595

Shiba, Y., Hirano, T., Nagashima, U., \& Ishii, K. 1998, J. Chem. Phys., 108,698

Shimanouchi, T. 1972, Tables of Molecular Vibrational Frequencies Consolidated, vol. I (National Bureau of Standards), 1

Suzuki, H., Yamamoto, S., Ohishi, M., et al. 1992, ApJ, 392, 551

Tachikawa, H. 1999, Phys. Chem. Chem. Phys., 1, 4925

Talbi, D., \& Ellinger, Y. 1996, Chem. Phys. Lett., 263, 385

Talbi, D., \& Ellinger, Y. 1998, Chem. Phys. Lett., 288, 155

Talbi, D., Ellinger, Y., \& Herbst, E. 1996, A\&A, 314, 688

Turner, B. E. 2001, ApJS, 136, 579

Pettersson, M., Lundell, J., Khriachtchev, L., \& Rasanen, M. 1998, J. Chem. Phys., 109, 618 